\title{
Spectroscopic observations of the eclipsing Polar MN Hya (RX J0929-24)
}

\author{
Gavin Ramsay ${ }^{1} \&$ Peter J Wheatley ${ }^{2}$ \\ ${ }^{1}$ Mullard Space Science Laboratory, University College London, Holmbury St.Mary, Dorking, Surrey, RH5 6NT \\ ${ }^{2}$ X-ray Astronomy Group, Dept of Physics 83 Astronomy, University of Leicester, University Road, Leicester, LE1 7RH
}

Accepted MNRAS July 1998

\begin{abstract}
We present low-medium resolution optical spectroscopy of the eclipsing AM Her system MN Hya (RX J0929-24). We determine the magnetic field strength at the primary accretion region of the white dwarf to be $42 \mathrm{MG}$ from the spacing of cyclotron features visible during $\phi \sim 0.4-0.7$. From spectra taken during the eclipse we find that the secondary has a M3-4 spectral type. Combined with the eclipse photometry of Sekiguchi, Nakada \& Bassett and an estimate of the interstellar extinction we find a distance of $\sim 300-700 p c$. We find unusual line variations at $\phi \sim 0.9$ : $\mathrm{H} \alpha$ is seen in absorption and emission. This is at the same point in the orbital phase that a prominent absorption dip is seen in soft X-rays.
\end{abstract}

Key words: binaries: eclipsing - stars: individual: MN Hya, RX J0929-24 - stars: magnetic fields - stars: variables

\section{INTRODUCTION}

MN Hya (RX J0929.1-2404) was discovered during the ROSAT all-sky survey and subsequently found to be an Polar (or AM Her) type of Cataclysmic Variable (Sekiguchi, Nakada \& Bassett 1994 and Buckley et al 1998a). Polars are interacting binary stars in which the white dwarf primary has a magnetic field strength sufficiently high to synchronise its spin period with the binary orbital period. Further, the magnetic field prevents the formation of an accretion disc and the accretion stream impacts directly onto the surface of the white dwarf.

MN Hya is one of 9 currently known eclipsing AM Her systems. These are particularly important systems to study since parameters such as the masses of the component stars can, in principle, be accurately determined. Of these systems, three (including MN Hya at $P_{\text {orb }}=3.39 \mathrm{hrs}$ ) are above the upper edge of the 'period gap' ( $\sim 2-3 \mathrm{hrs})$. MN Hya also shows two 'dips' in soft X-rays where the accretion stream obscures emission from the hot post shock region above the white dwarf (Buckley et al 1998b). The deepest dip occurs at $\phi \sim 0.9$ (just before the eclipse of the white dwarf by the secondary star), while another less deep dip occurs at $\phi \sim 0.5$. These dips are not seen in the optical continuum (Sekiguchi, Nakada \& Bassett 1994).

We have obtained low-medium resolution phase resolved optical spectroscopy of MN Hya and determined the spectral type of the secondary star, its distance, the mag- netic field strength of white dwarf and examine how the optical spectrum varies through the absorption dips.

\section{OBSERVATIONS AND REDUCTION}

Spectra were obtained using the ESO $3.6 \mathrm{~m}$ telescope at La Silla, Chile on 1997 Feb 7/8 \& 8/9. The conditions were photometric and the seeing was between $1.0-1.5^{\prime \prime}$. On the first night alternate images (with exposures typically 300 sec) were taken with the R300 (6000-9800 $\AA$ ) and B300 (3800-6800 $\AA$ ) grisms. With a slit of $1.5^{\prime \prime}$ the resolution in both grisms was $\sim 15 \AA$. On the second night, only the B300 grism was used and the exposures were 180 sec. Wavelength calibration of the flat-fielded and bias-subtracted twodimensional images was performed using a He-Ar arc spectra taken at the start and end of the night (flexure is negligible on ESO $3.6 \mathrm{~m}$ telescope). The wavelength calibrated spectra were flux calibrated using the standard stars GD 108 and Feige 67.

\section{THE STRENGTH OF THE MAGNETIC FIELD OF THE WHITE DWARF}

One method of determining the magnetic field strength of the white dwarf is from the spacing of cyclotron harmonics. For the field strengths seen in Polars $(B \sim 10-200 \mathrm{MG})$ cyclotron harmonics are seen as broad humps in optical/IR 
spectra. The cyclotron flux originates from electrons spiraling around the field lines in the post-shock region above the surface of the white dwarf. These humps vary in their intensity and (to a lesser degree their wavelength) as a function of our viewing angle to the post-shock region (ie they vary over the orbital phase).

There was no obvious sign of cyclotron features in our optical spectra. To make a more detailed search for such features we made a mean 'blue' and 'red' spectrum: the mean blue spectrum was made up of 'blue' spectra (3800-6800 ̊) taken on the second night, while the mean red spectrum was made up of 'red' spectra (6000-9800 $)$ taken on the first night (on the second night no red spectra were obtained). We then averaged spectra to give spectra covering the phase range $\phi=0.05-0.15,0.15-0.25$ etc and then normalised these spectra by dividing by the appropriate mean spectrum (we use the ephemeris of Buckley et al 1998a which defines $\phi=0.0$ as the center of the eclipse of the white dwarf by the secondary). Figure 1 shows these normalised spectra over the binary orbit. There is no evidence for cyclotron features until the phase interval covering $\phi=0.35-0.45$ when humps are seen at $\sim 5800 \& 7200 \AA$. These continue to be seen until $\phi \sim 0.7$. This is confirmed if we divide the spectrum covering $\phi=0.35-0.45$ by the spectrum covering $\phi=0.05-0.15$.

To determine the wavelength of cyclotron harmonics for a given magnetic field strength $B$, we write $\omega_{B}=e B / m_{e} c$ (where $\omega_{B}$ is the fundamental cyclotron frequency) as $\omega_{B}=$ $1.76 \times 10^{14} B_{7}$ (where $B_{7}=B / 10^{7} \mathrm{G}$ ). We then use equation (3) of Cropper et al (1988) and put it in terms of $\lambda$ :

$\frac{\omega}{\omega_{B}}=\frac{-1+\sqrt{1+\left(8 n \sin ^{2} \theta / \mu\right)}}{\left(4 \sin ^{2} \theta / \mu\right)}=\frac{\lambda_{B}}{\lambda}$

where $\mu=m_{e} c^{2} / k T=511.1 / T, T$ is in units of $\mathrm{keV}, n$ is the cyclotron harmonic number and $\theta$ is the viewing angle to the magnetic field angle. We can now rearrange the above as a function of $\lambda$ :

$\lambda=\frac{4.28 \times 10^{5} \sin ^{2} \theta}{\mu B_{7}\left(-1+\sqrt{1+\left(8 n \sin ^{2} \theta / \mu\right)}\right)}$

where $\lambda$ is in $\AA$.

To determine the magnetic field strength we searched a range of parameters which gave consecutive harmonics placed at $\sim 5800 \& 7200 \AA$. For lower values of $\theta$ the harmonics are blue shifted by a small amount compared to higher values. For low magnetic fields $(B \sim 15 \mathrm{MG})$ the individual harmonics become indistinguishable and form a continuum. Similarly for temperatures $>15 \mathrm{keV}$ individual harmonics are not visible. By comparing the expected wavelength of the harmonics by eye to the observed spectrum, we found that $B=42 \mathrm{MG}, k T=9 \mathrm{keV}$ and $\theta=90^{\circ}$ gave the best 'fit'. Assuming that we have correctly identified harmonics at $\sim 5800 \&$ $7200 \AA$, we estimate the error on $B$ to be $\sim 1-2 \mathrm{MG}$ based on our search of the variable parameters.

The value of $B=42 \mathrm{MG}$ found here compares with $B \sim 20 \mathrm{MG}$ estimated by Buckley et al (1998a) from a single spectrum of exposure $3000 \mathrm{sec}$. Their spectrum was not flux-calibrated and they caution that the small-scale variations seen in their spectrum could have been due to other factors rather than cyclotron humps. We therefore consider that our estimate of $B=42 \mathrm{MG}$ to be more reliable. We will examine this in more detail in $\S$.

\section{THE SECONDARY STAR AND ITS DISTANCE}

We obtained a red spectrum of MN Hya which was centred on the eclipse (figure 8). Although emission lines are visible, this may be due to some pre or post eclipse flux being recorded. Absorption features due to the secondary star are visible at $7150 \AA$ and $8450 \AA$. However, the low resolution of the spectrum did not permit a detailed analysis of these features. No secondary features are visible outwith the eclipse.

To determine the spectral type of the secondary star we compared its spectrum taken during the eclipse with late type spectra kindly supplied by Dr Robert C Smith. These are overlaid in figure 2. The depth of the TiO band at 8450 $\AA$ indicates that it is later than M1, while the lack of a prominent Na I feature at $8183-8194 \AA$ suggests that it is earlier than $\sim \mathrm{M} 6$. Bearing in mind that some flux has been recorded which is not due to the secondary (since we observe emission lines), the overall spectral shape is closest to M3.5.

Using the empirical mass-radius relationship of Patterson (1984), the mass of the secondary is expected to be 0.31 $M_{\odot}$ for an orbital period of $P_{\text {orb }}=3.39 \mathrm{hrs}$. Using the tables of spectral type against stellar mass in Zombeck (1990), we find a spectral type of $\sim \mathrm{M} 3$. Within the uncertainties involved this is consistent with the spectral type determined from the eclipse spectrum.

Using the photometry of Sekiguchi, Nakada \& Bassett (1994), where they find $m_{I c}=18.5$ at mid-eclipse, and the absolute magnitude of an M3 V star $\left(M_{I}=8.71\right.$ : Bessell 1991), we obtain a distance of $910 \mathrm{pc}$ if zero absorption is assumed. However, the X-ray data obtained using ROSAT show that the absorption to MN Hya is $\sim 2.5 \times 10^{20} \mathrm{~cm}^{-2}$ (Buckley et al 1998b). This implies $A_{I}=0.9$ and a distance of 610 pc. For a M4 V star $\left(M_{I}=9.85\right.$ : Bessell 1991) we get distance of 360 and $540 \mathrm{pc}$ assuming $A_{I}=0.9$ and $A_{I}=0.0$ respectively. We estimate that the distance to MN Hya is between $300-700$ pc.

\section{LINE INTENSITY VARIATIONS}

\subsection{Spectra covering the absorption dip}

In the previous sections we have concentrated on broad spectral features. Here we examine individual line variations over the course of the orbital period. Perhaps the most interesting feature is the interval immediately before the eclipse ( $\phi \sim 0.90)$ which coincides with the most prominent dip seen in soft X-rays (Buckley et al 1998b). Figure 3 shows spectra starting at $\phi=0.85$ where the spectrum has strong Balmer and He II line emission and is typical of a Polar. However, at $\phi=0.87$ the emission lines start to become less prominent, especially towards bluer wavelengths. At $\phi=0.89$ only 5 emission lines are present - HeII $\lambda$ 4100, H $\delta$, HeI $\lambda 4388$, $\mathrm{HeII} \lambda 4686, \mathrm{H} \beta$ and $\mathrm{H} \alpha$. Moving later in phase, at $\phi=0.90$ and 0.92 , we find that HeII $\lambda 4686$ is still in emission, while $\mathrm{H} \alpha$ and $\mathrm{H} \beta$ appear in absorption and emission. At this phase the emission and absorbed components of the $\mathrm{H} \alpha$ line have red shifted radial velocities of $\sim 270 \mathrm{~km} \mathrm{~s}^{-1}$ and $\sim 870 \mathrm{~km}$ $\mathrm{s}^{-1}$ respectively.

Later in phase, at $\phi=0.94$ and 0.96 , very broad emission lines are seen shortwards of $5000 \AA$, although HeII $\lambda 4686$ is as narrow as before. In the spectrum taken immediately 


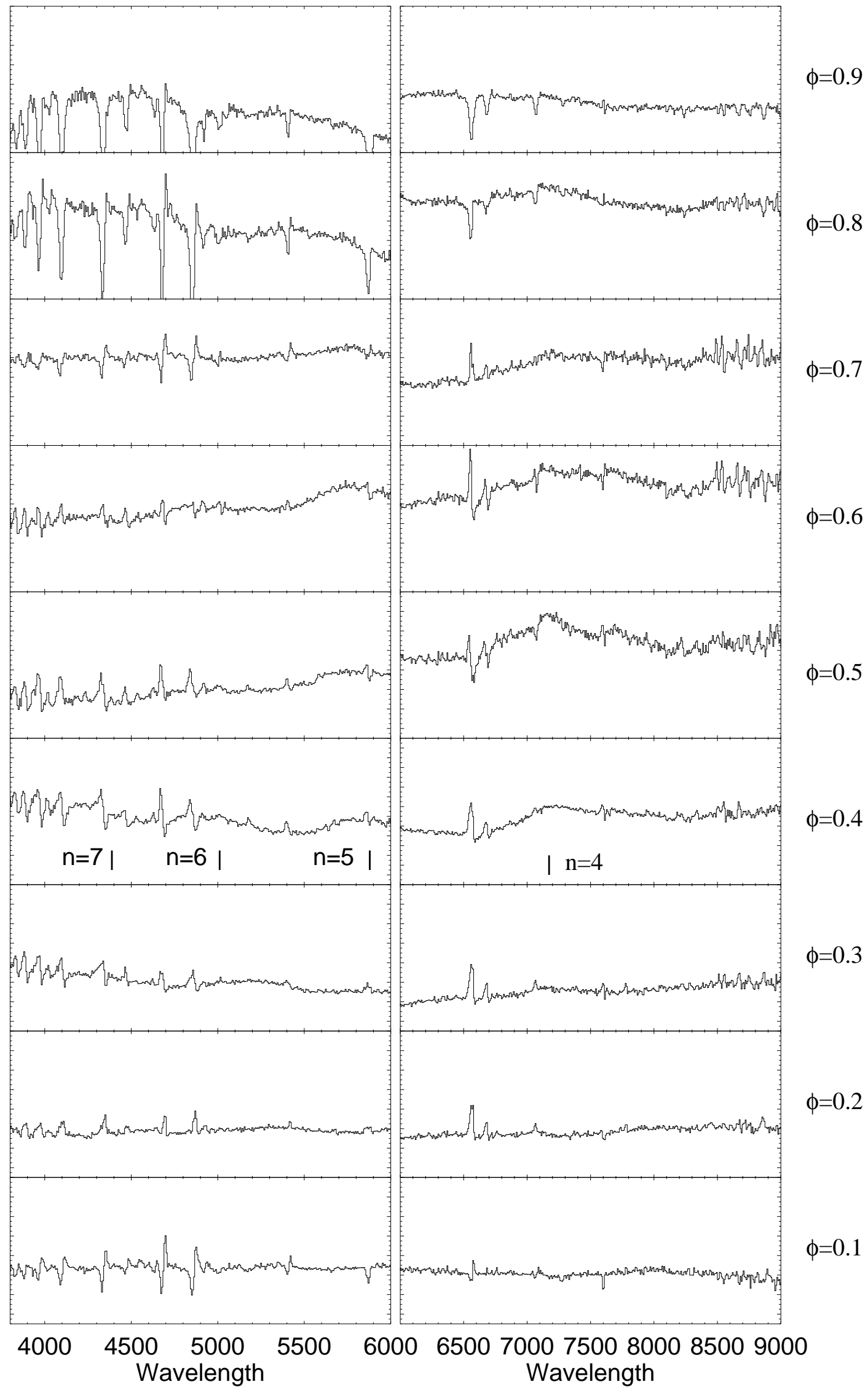

Figure 1. The phase averaged normalised spectra covering the binary orbital cycle (the eclipse phase is shown to the right). Averaged blue and red spectra covering 0.1 of the orbital cycle were obtained and then divided by the mean blue and red spectrum respectively. (For example the bin $\phi=0.1$ covers $\phi=0.05-0.15$ ). The unusual line profiles are simply due to radial velocity variations over the orbital cycle. The vertical axis covers the same interval range. The wavelengths of harmonic numbers $n=4-7$ are shown for a temperature of $9 \mathrm{keV}$, a magnetic field strength of $42 \mathrm{MG}$ and viewing angle of $90^{\circ}$.

(c) 0000 RAS, MNRAS 000, 000-000 


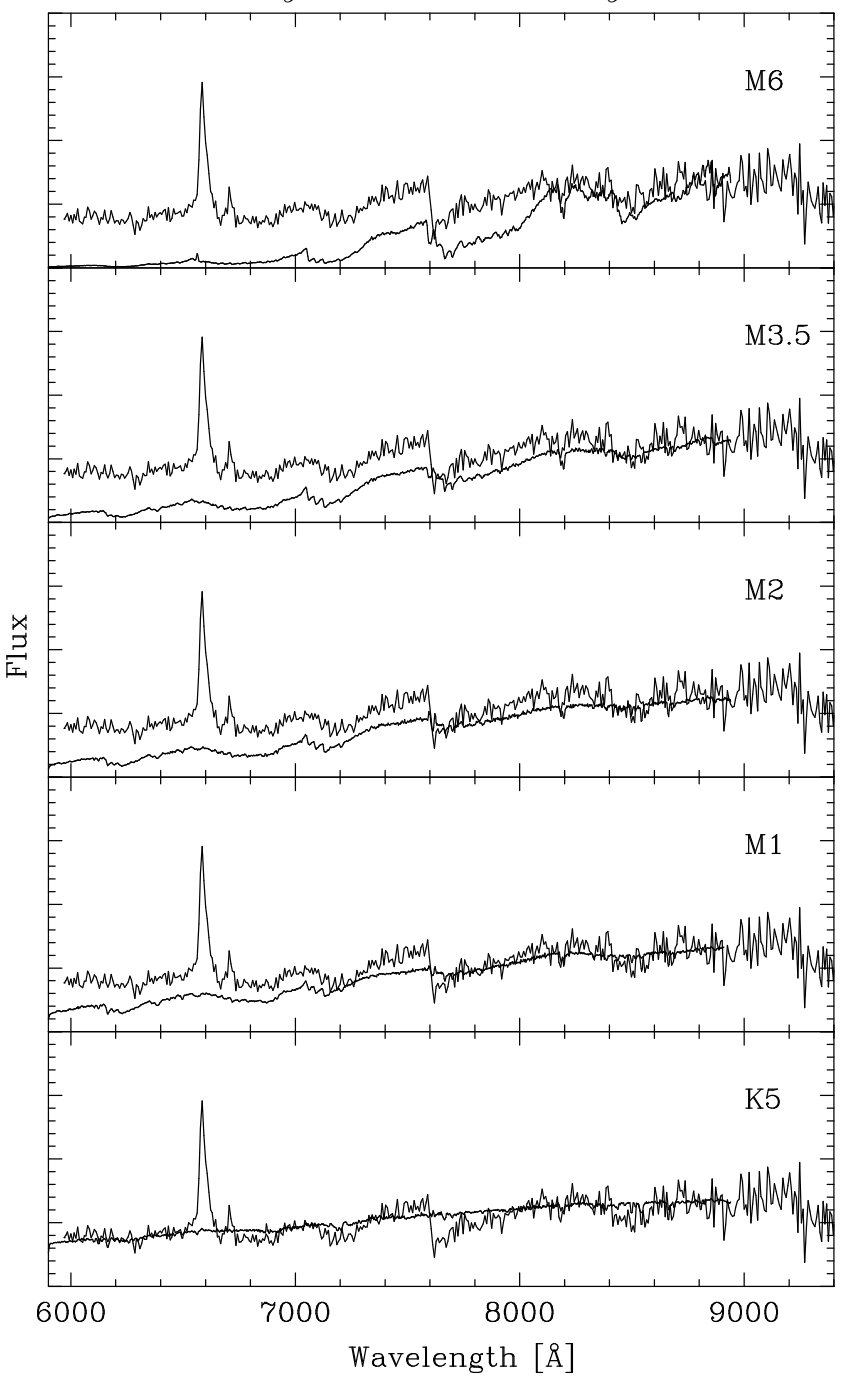

Figure 2. The eclipse spectrum of MN Hya (which contains emission lines probably due to some pre-eclipse flux being recorded) overlaid with various spectral types which were kindly supplied by Dr Robert C Smith.

before eclipse, HeI $\lambda 5876$ is back in absorption and all other lines apart from HeII $\lambda 4686$ are very weak. By $\phi \sim 0.05$, the narrow component of the lines has returned. In $\S 6$ we discuss the interpretation of these dip spectra while in the next section we examine the line profile variations over the whole of the orbital period in more detail.

\subsection{Fitting the line profiles}

We fitted the emission lines using a single Gaussian profile for the Helium lines and two Gaussian profiles in the case of the Hydrogen lines (there were some spectra where an additional Gaussian improved the fit to the He lines, but not in any consistent manner). The line-flux was determined by simply summing the flux under the Gaussian(s). The lineflux variations of $\mathrm{H} \alpha, \mathrm{H} \beta$, $\mathrm{HeI}$ (5876) and HeII (4686) are shown in the top panel of figure 4 . (We only show the results from the night of Feb 8/9 since on the previous night the sampling was lower). The radial velocities and EW for the narrow and broad components of $\mathrm{H} \alpha, \mathrm{H} \beta, \mathrm{HeI}(5876)$ and HeII(4686) are shown in figure 4 .

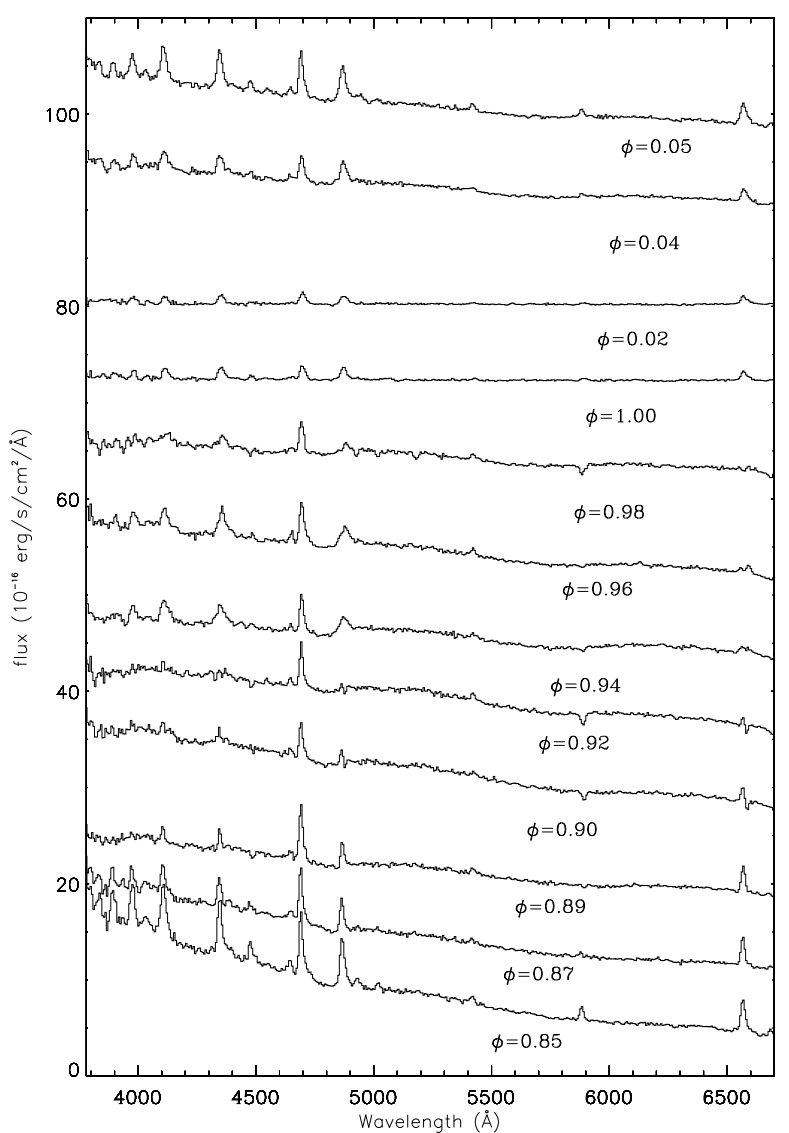

Figure 3. Spectra starting at $\phi=0.86$ are shown indicating how the spectrum changes during the course of the absorption dip. Spectra have been offset by 12 flux units vertically.

\begin{tabular}{lllc}
\hline Component & $\gamma\left(\mathrm{km} \mathrm{s}^{-1}\right)$ & $K\left(\mathrm{~km} \mathrm{~s}^{-1}\right)$ & $\phi_{o}$ \\
\hline $\mathrm{H} \alpha$ (narrow) & $102 \pm 29$ & $134 \pm 43$ & $-0.24 \pm 0.04$ \\
$\mathrm{H} \alpha$ (broad) & $91 \pm 143$ & $732 \pm 176$ & $-0.36 \pm 0.03$ \\
$\mathrm{H} \beta$ (narrow) & $66 \pm 35$ & $259 \pm 52$ & $-0.28 \pm 0.03$ \\
$\mathrm{H} \beta$ (broad) & $102 \pm 75$ & $831 \pm 115$ & $-0.34 \pm 0.02$ \\
\hline
\end{tabular}

Table 1. The radial velocity parameters for the $\mathrm{H} \alpha$ and $\mathrm{H} \beta$ lines fitted using two Gaussian profiles. $K$ is the velocity amplitude and $\phi_{o}$ is the phase at which the observed radial velocity equals the $\gamma$ velocity when crossing from blue to red. The errors are quoted at the 90 per cent confidence level.

The narrow and broad components were fitted with a sinusoidal function of the form:

$$
V(\phi)=\gamma+K \sin \left(2 \pi\left(\phi-\phi_{o}\right)\right)
$$

where $V(\phi)$ is the radial velocity at phase $\phi, \gamma$ is the component of the accretion streams velocity perpendicular to the orbital plane projected along the line of sight, $K$ is the velocity amplitude and $\phi_{o}$ is the phase at which the observed radial velocity equals the $\gamma$ velocity when crossing from blue to red. To estimate the errors, the velocity error was adjusted to normalise the reduced $\chi_{\nu}^{2}$ to 1.0 since it was not possible to derive meaningful errors to the Gaussian fits. Table 1 shows the best fit parameters and the 90 per cent confidence interval while figure 4 shows the best fits along with the data. We now discuss the implications these results have on our view of the accretion stream. 

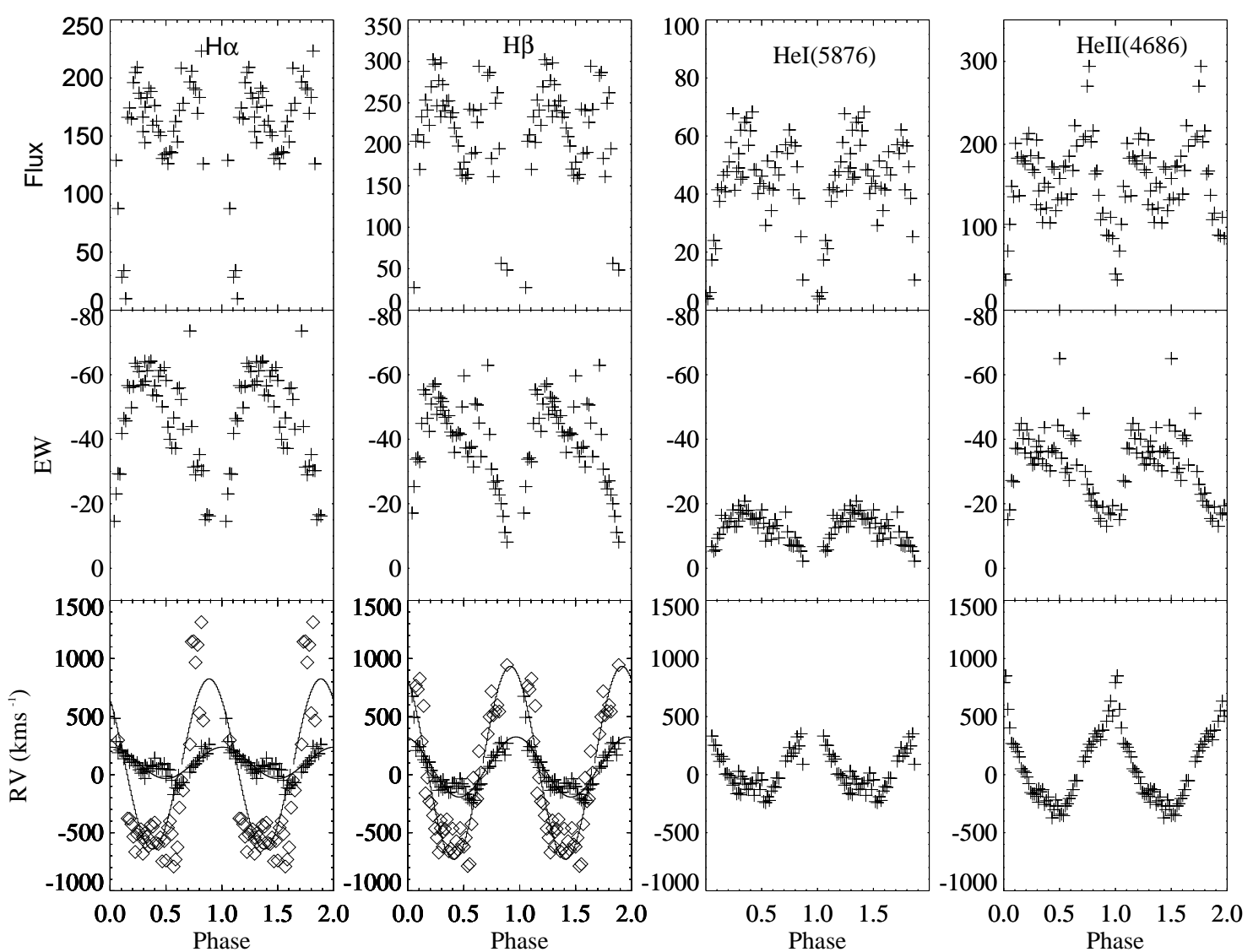

Figure 4. The line fluxes $\left(10^{-16} \mathrm{erg} \mathrm{s}^{-1} \mathrm{~cm}^{-2}\right)$, radial velocities $\left(\mathrm{km} \mathrm{s}^{-1}\right)$ and $\mathrm{EW}$ variations for the $\mathrm{H} \alpha, \mathrm{H} \beta, \mathrm{HeI}(5876)$ and $\mathrm{HeII}$ (4686) lines over the binary orbital period. For the $\mathrm{H} \alpha$ and $\mathrm{H} \beta$ lines we were able to distinguish a narrow and broad component. We show their combined flux and EW while we show the radial velocity of the broad component as a diamond and the narrow component is shown as a plus in the lower panel.

\section{THE ACCRETION STREAM}

We have been able to distinguish two components in the Hydrogen line profiles: a narrow and a broad component. The broad component is most probably due to emission originating from the accretion stream close to the white dwarf because of its high maximum radial velocity. Since the stream initially has the rotational velocity of the secondary star, the broad component will be seen with maximum red shift before $\phi=0.0$ - in this case at $\phi \sim 0.91$.

The narrow component has a much lower radial velocity than the broad component: if the narrow component originated from the heated face of the secondary star we would expect to see it with maximum red shift at $\phi \sim 0.25$. The fact that we see it with maximum red shift at $\phi \sim 0.98 \mathrm{im}-$ plies that the narrow component which we can distinguish is not a good marker of the secondary star. In non-eclipsing systems, where there is no extra information on the orbital phase of the secondary, it has often been assumed that the narrow component originates from the irradiated face of the secondary. In the case of MN Hya we know that the maximum red-shift of the narrow component occurs close to the eclipse, so cannot be emitted by the secondary. Instead the accretion stream close to the secondary is the most likely candidate and this view is supported by the fact that the narrow component is eclipsed and with a duration longer than the white dwarf eclipse. We note that this may well be true for most/all systems, and that low-resolution spec- troscopy is not a suitable method for detecting the motion of the secondary (and thus measuring the mass ratio of the system). Schwope, Mantel \& Horne (1997), for instance, show that with higher resolution spectroscopy, it is possible to separate the narrow components emitted by the stream and the secondary.

There are a number of systems which show dips in soft $\mathrm{X}$-rays which have been attributed to the accretion stream obscuring the hot post shock region above the white dwarf (eg Watson et al 1989, Buckley et al 1998b). However, there is only 1 other Polar in which optical lines appear in absorption during a stream dip (EF Eri: Verbunt et al 1980, Allen, Ward \& Wright 1981). In the case of EF Eri, lines redder than $5500 \AA$ appeared in absorption. In the case of MN Hya we find that at $\phi \sim 0.90$ the $\mathrm{H} \alpha$ and $\mathrm{H} \beta$ lines are seen in both emission and absorption, both of which are redshifted. The emission and absorption components of the $\mathrm{H} \alpha$ line have radial velocities of $\sim 270 \mathrm{~km} \mathrm{~s}^{-1}$ and $\sim 870 \mathrm{~km} \mathrm{~s}^{-1}$ respectively. Since the stream is curved and we do not know where the emission and absorption components originate we cannot determine the true velocities of these components. It is, however, likely that the absorption component originates closer to the white dwarf than the emission component. On the other hand, the fact there are two accreting poles (cf next section) and hence two accretion streams once the stream attaches onto the magnetic field lines of the white dwarf, may complicate this interpretation. 


\section{ARCHIVAL POLARISATION DATA}

In $\S 3$ we presented phase resolved optical spectra of MN Hya which show features which we interpret as cyclotron humps and estimate a magnetic field strength of the white dwarf to be $B=42 \mathrm{MG}$ from their spacing. This is higher than the $B \sim 20 \mathrm{MG}$ estimated by Buckley et al (1998a) from an integrated non-flux calibrated spectrum. Buckley et al then modeled their white light optical polarimetry using $B=20$ MG. To test if their same polarimetry data can be adequately modeled using a field strength of $B=42 \mathrm{MG}$ and determine the location of the accretion regions on the surface of the white dwarf we measured the data points from Fig. 4 of Buckley et al (1998a) and fitted their data using the optimisation method of Potter, Hakala \& Cropper (1998). Up until now most polarimetry data, including that of Buckley et al, have been modeled by constructing accretion regions on the surface of the white dwarf by hand and then a good fit to the data was achieved by trial and error. The method of Potter, Hakala \& Cropper finds the best fit in an objective manner. In our new fit we fixed the magnetic field strength at $B=42 \mathrm{MG}$ and the inclination at $75^{\circ}$ (derived from the eclipse duration, Buckley et al 1998a).

The fits of Buckley et al (1998a) implied that two accretion regions are visible (the main region being partially visible near the lower magnetic pole) and that they are greatly extended in magnetic longitude (the secondary region is $300^{\circ}$ in length and is near the upper pole). Both accretion regions were offset from their respective magnetic poles by $\sim 20^{\circ}$. Our fits to the same polarimetric data are shown in figure 5. The fits to the intensity data (white light) and the circular polarimetry are good and better than the fit obtained by Buckley et al (1998a). The level of linear polarisation is rather low and the peak in linear polarisation around the eclipse phase is almost certainly due to instrumental or sky background effects in the original data. The best fit to the data was obtained with the magnetic axis having a tilt of $\theta_{d}=30^{\circ}$ with respect to the spin axis of the white dwarf. Further, our fit implies accretion regions which are less extended than found by Buckley et al: the main region is extended by less than $60^{\circ}$ in magnetic longitude and is offset from the magnetic pole by $15^{\circ}$ and is visible on the limb of the white dwarf between $\phi \sim 0.45-0.75$. The secondary region (which is much less bright than the primary region) is roughly circular in shape and is visible between $\phi \sim 0.65-0.25$ and comes almost face on at $\phi=0.9$.

The location of the primary accretion region is consistent with the detection of cyclotron humps in the optical spectra between $\phi \sim 0.35-0.65$. The fact that no cyclotron features are seen when the secondary accretion region is visible may simply be due to the features being too faint to detect or that the magnetic field at the secondary region is lower than the primary accretion region. Buckley et al (1998b) suggested that the latter may account for the higher soft to hard X-ray ratio over $\phi=0.03-0.45$ compared to $\phi=0.45-0.87$.

We conclude that our estimate of $B=42 \mathrm{MG}$ is consistent with the optical polarimetry and that at $\phi \sim 0.9$ it is the secondary accretion region which is obscured by the accretion stream.

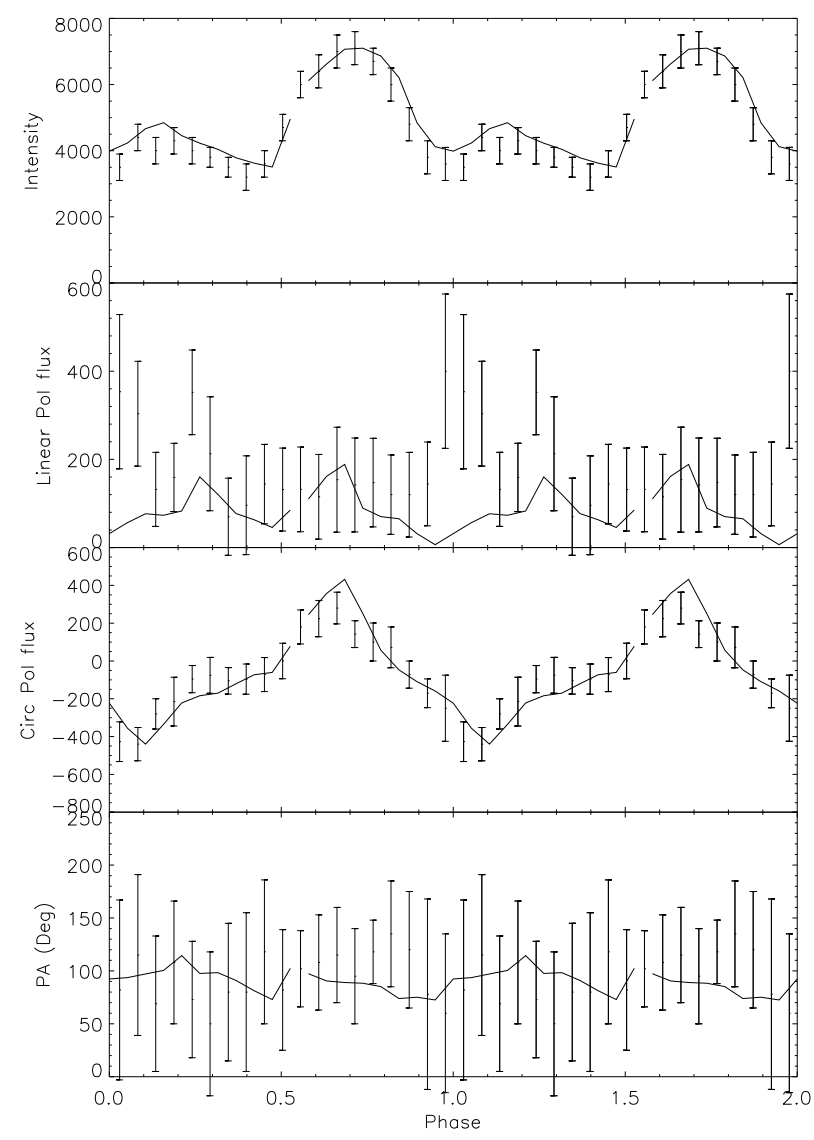

Figure 5. The white light polarimetry data presented in Buckley et al (1998a) - from top to bottom: intensity, linearly polarised flux, circularly polarised flux and the position angle. The best fits using the optimisation method described by Potter, Hakala \& Cropper 1998 are overlaid - the inclination was fixed at $75^{\circ}$, the magnetic field was fixed at $42 \mathrm{MG}$.

\section{ACKNOWLEDGMENTS}

This paper is based on observations obtained at the European Southern Observatory, La Silla, Chile using the ESO $3.6 \mathrm{~m}$ telescope and we would like to thank ESO for their support during our observing period. PJW is supported by a PPARC post-doctoral fellowship. We are grateful to Dr Stephen Potter for modelling the photo-polarimetric data of Buckley et al and Dr Robert C. Smith for supplying us with spectra of late-type stars. Further, thanks go to Dr David Buckley for suppling the eclipse ephemeris and pre-prints before publication.

\section{REFERENCES}

Allen, D. A., Ward, M. J., Wright, A. E., 1981, MNRAS, 195, 155 Bessell, M. S., 1991, AJ, 101, 662

Buckley, D. A. H., Ferrario, L., Wickramasinghe, D. T., Bailey, J. A., 1998a, MNRAS, 295, 899

Buckley, D. A. H., Barrett, P. E., Haberl, F., Sekiguchi, K., 1998b, submitted, MNRAS

Cropper, M. et al 1989, MNRAS, 236, 29P

Frank, J., King, A. R., Raine, D. J., 1992, Accretion Power in Astrophysics, Cambridge Univ Press, Cambridge

Patterson, J., 1984, ApJS, 54, 443 
Potter, S. B., Hakala, P. J., Cropper, M., in press, MNRAS

Sekiguchi, K., Nakada, Y., Bassett, B., 1994, MNRAS, 266, L51

Schwope, A. D., Mantel, K.-H., Horne, K., 1997, A\&A, 319, 894

Verbunt F., Heuvel E. P., van der Linden T. J., Brand J., van Leeuwen F., van Paradijs J., 1980, A\&A, 86, L10

Zombeck, M. V., 1992, Handbook of Space Astronomy \& Astrophysics, 2nd edition, CUP 